\title{
Laser Printing of Organic Electronics and Sensors
}

\author{
Ioanna Zergioti \\ National Technical University of Athens, Physics Department, Iroon Polytehneiou 9, 15780, Zografou, Athens, Greece.
}

zergioti@central.ntua.gr

This work presents the use of the Laser-Induced Forward Transfer (LIFT) process as an advanced tool for organic electronics and biosensor fabrication. LIFT is an alternative micro-patterning technology that employs pulsed laser irradiation for the selective deposition of a liquid or solid target material onto a receiving substrate. LIFT) is a non-contact direct printing method that can deposit patterns of functional materials, in liquid or in solid phase, including organic, carbon-based nanomaterials, biological compounds, metal nanoparticles, and dielectrics on different substrates including flexible materials. More specifically, the LIFT process is used for direct printing of Agbased nanoparticles, organic semiconducting materials for organic transistors and biomaterials (enzymes and photosynthetic proteins) for environmental biosensor fabrication. The use of LIFT has shown the possibility of direct immobilization of biomaterials onto the surface of the sensors without the use of chemical linkers.

DOI:10.2961/jlmn.2013.01.0007

Keywords: Laser-Induced Forward Transfer, LIFT, sensors, Ag laser printing

\section{Introduction}

Currently there is an industrial need for digital printing techniques, due to the increasing interest in microelectronic devices. Therefore, many direct-write techniques such as ink-jet printing, dip-pen lithography and laser printing have been developed to fulfill the required technological need.

Ink-jet printing is a particularly attractive direct-write technique, for surface patterning and the delivery of precise quantities of materials. Nozzles used by ink-jet printing setups require specific parameters for the viscosity of the solutions used which in turn can impose limitations on the spatial resolution achievable. For example, the typical droplet volume from commercially available inkjet nozzles is of the order of several picoliters, resulting in individual pixel sizes of 30 to $50 \mu \mathrm{m}$ but which have poor thickness uniformity and surface morphology $[1,2]$.

Dip-pen lithography is another direct-write technique which is based on the use of an ink-coated AFM tip. However, this technique involves direct-contact between tip and substrate, and hence this can affect specific substrate properties.

Laser-Induced Forward Transfer (LIFT), on the other hand, is a process that can overcome these limitations. It is a non-contact direct printing method that can deposit patterns of functional materials, in liquid or in solid phase, including metal nanoparticles, dielectrics and a great variety of biomaterials onto different kinds of substrates which include flexible materials.

In this work, we present the direct printing process of organic semiconducting and metallic nanoparticles for the fabrication of organic thin film transistors (TFTs) as well as photosynthetic materials and oligonucleotide sequences for the fabrication of label-free biosensors using the technique of LIFT.

Organic thin-film transistors (OTFTs) have recently received considerable attention because of their potential applications in flexible, low-cost integrated circuits. Typical examples include smart cards and radio frequency identification (RFID) tags, display backplanes such as liquid crystal displays and electronic paper, and organic electroluminescent displays. However, the widely-adopted photolithographic and electro-less deposition techniques for manufacturing the conductive circuits of the printed circuit boards are time consuming, very complicated and expensive.

Photosynthetic materials are used for environmental sensors, because photosynthesis is an electron transfer procedure. Their immobilization onto the electrodes surface can involve both physical and chemical procedures $[3,4]$, but in terms of simplicity and photosynthetic material vitality, physical procedures are considered as the ideal immobilization method. However, the presence of an additional functionalization step in the fabrication of biosensors - usually both time consuming and requiring special care in handling the hazardous chemicals involved is one of the main disadvantages of chemical immobilization methods. In our approach, we have adopted LIFT processing [5] as an advanced tool for direct immobilization of photosynthetic material onto low cost non-functionalized gold screen-printed electrodes (SPEs). In this work, photosynthetic thylakoid membranes were extracted from Spinacea oleracea and printed in the liquid phase onto non-functionalized gold SPE electrodes using LIFT. The high kinetic energy of the transferred material induces a correspondingly high impact pressure and enhances physical adsorption onto the electrode surface. As a result, high photocurrent activity was achieved while using extremely low quantities of thylakoid membranes (0.61 $\mu \mathrm{g}$ of chlorophyll).

Finally, there is also specific interest in DNA biosensors because of the unique ability of the oligonucleotides to detect specific target sequences. Therefore, DNA sensors not only permit multi-sequence detection, but also reduce the analysis time since the hybridization of DNA sequences is a very fast procedure.

\section{Experimental}


The LIFT experiments were carried out using single pulses from a frequency quadrupled $\mathrm{Nd}$ :YAG laser (10 ns pulse duration), operating at $266 \mathrm{~nm}$. The laser beam was expanded to irradiate a variable diameter circular or rectangular mask in order to select a homogeneous part of the beam, and was then imaged by a converging lens onto the coated side of the donor. The donor substrate was a $25 \mathrm{~mm}$ diameter quartz plate coated with a $40 \mathrm{~nm}$ thick titanium (Ti) laser-absorbing layer.

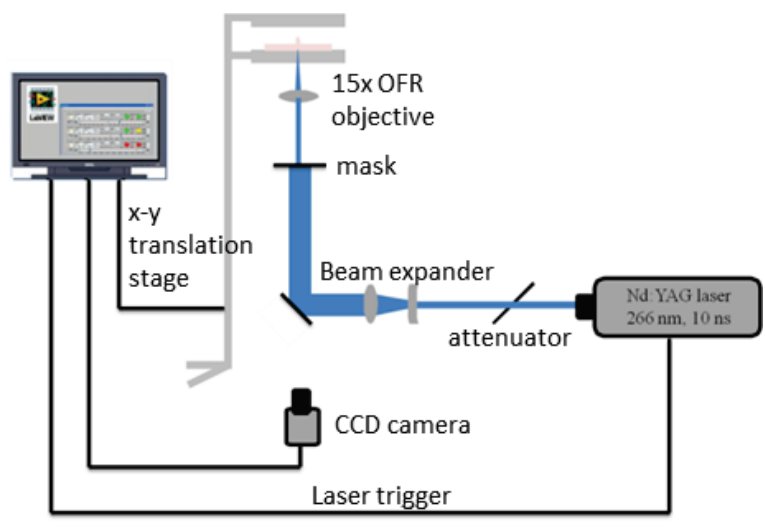

Fig. 1 Schematic illustration of the LIFT printing experimental setup.

\section{Results and discussion \\ 3.1 Ag lines}

It has been demonstrated that liquid transfer via LIFT can be used to deposit individual spots (voxels) of various liquids (such as nanoparticle (NP) inks) on a substrate regardless of the physical properties of the liquid. The ability to print even viscous inks makes LIFT a more versatile method than traditional ink-jet printing. When irradiated by a short pulse the liquid on the donor substrate is propelled towards the receiver substrate in the form of a stable jet [6]. When the jet reaches the receiver substrate a 'bridge' that facilitates the transfer of the material is formed between the donor and receiver substrates. The resulting voxels remain in a liquid state after the transfer but are also fairly immobile. Nevertheless there are several disadvantages when using this method including a wellknown problem associated with ink-jet printing namely the stability of a liquid-printed line [7]. When printing continuous lines the jet comes in contact with the previously printed material and this interaction together with the substrate movement can cause instabilities, creating bulges (bubbles) along the length of the printed lines. The main factor for the creation of these instabilities is the advancing contact angle of the liquid with the substrate.

$\mathrm{Ag}$ lines were printed on silicon or flexible substrates to be used as electrodes. These electrodes were fabricated by means of liquid phase Ag nanoparticles printing. The ink used in these experiments was the commercially available SunTronic Jettable Silver U5603, with viscosity 10-13 cps at $25{ }^{\circ} \mathrm{C}$, with a $20 \%$ silver content, an average nanoparticle size of $80 \mathrm{~nm}$ and a surface tension between 27-31 dynes $/ \mathrm{cm}$. Several solutions were prepared with different viscosities by water dilution, and were spin coated on
quartz/Ti substrates. The donor substrates were weighed before and after the spin coating of the Ag nanoparticle ink. With the weight and density of the deposited material known, its volume was calculated and since the surface area of the donor substrate was also known the thickness of the deposited film was estimated at about $5 \mu \mathrm{m}$. The thicknesses of all the samples were the same to facilitate investigation of the effect of the viscosity in the printing process. Different fluences were used in the printing process for each solution in order to optimize the results. Dots were printed to examine the resulting volume of the voxel thereby configure the printing process for the lines. In order to achieve continuous lines a $30-40 \%$ overlap of the printed spots (voxels) was used, and lines of $1-2 \mathrm{~mm}$ in length were printed and characterized both morphologically and electrically. Our recent results include the printing of interdigitated electrodes to be used in OTFTs.

The resulting lines from the various solutions prepared demonstrated visible differences in their end quality. Diluting the Ag NP ink with 40-50\% water was found to give the best results morphologically: the borders of the lines were well defined and they were relatively bubble free. All the samples were annealed after deposition to evaporate the solvent, harden the electrodes and sinter the nanoparticles to increase their conductivity. The sintering conditions used were one hour at $150{ }^{\circ} \mathrm{C}$ for $\mathrm{SiO}_{2}$ substrates and one hour and a half at $120{ }^{\circ} \mathrm{C}$ for polyethylene naphthalate (PEN) substrates.

The thickness of the printed electrodes was measured using a profilometer. Several measurements of each line were taken in order to find the mean value of the thickness for the lines. The results are shown in Table 1 along with the resistivity measurements from the same electrodes.

Table 1. Thickness and Resistivity of electrodes as a function of Ag NP ink volume in the solution

\begin{tabular}{|c|c|c|}
\hline $\begin{array}{c}\text { Ag NP ink } \\
\text { volume }\end{array}$ & Thickness (nm) & $\begin{array}{c}\text { Resistivity } \\
(\mathbf{\Omega c m})\end{array}$ \\
\hline $40 \%$ & 111 & $6.48 \times 10^{-5}$ \\
\hline $50 \%$ & 119 & $4.78 \times 10^{-5}$ \\
\hline $65 \%$ & 87 & $4.32 \times 10^{-5}$ \\
\hline $80 \%$ & 111 & $4.56 \times 10^{-5}$ \\
\hline $100 \%$ & 94 & $3.31 \times 10^{-5}$ \\
\hline
\end{tabular}

In Fig. 2a individual spots of silver NP ink are shown before annealing and were all printed using the same laser fluence in order to test the repeatability of the printing. Several different arrays of spots were printed to help us identify the ideal conditions for printing the lines. The diameter of the voxels is seen to be approximately $30 \mu \mathrm{m}$. In Fig. $2 \mathrm{~b}$ annealed lines (electrodes) using the $50 \% \mathrm{Ag}$ NP ink solution are presented. Note that the "bubble effect" is significantly reduced. 
a)

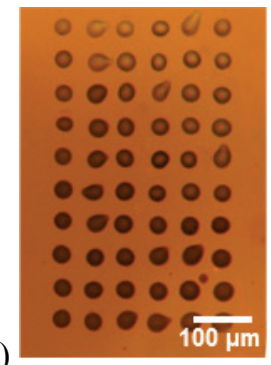

Fig. 2 a) Spots (voxels) of Ag NP ink printed using LIFT b) $\mathrm{Ag}$

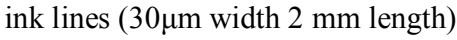

\subsection{Organic thin film transistors (OTFTs)}

Previous work has demonstrated that high quality gate and Source/Drain (S/D) Ag nano-ink electrodes for OTFTs can be successfully fabricated via laser printing [8].The successful laser printing of both $\mathrm{Ag} \mathrm{S} / \mathrm{D}$ electrodes and a semiconducting organic layer resulting in operative OTFT devices [9] has also been reported previously.

Here, we report the realization of OTFTs with a bottom contact (BC) configuration where both the organic semiconductor (P3HT) and the Ag nano-inks S/D electrodes have been printed via LIFT. The receiver substrate was a silicon (Si) wafer oxidized by a $300 \mathrm{~nm}$ thick layer of silicon dioxide $\left(\mathrm{SiO}_{2}\right)$ and used as the gate and dielectric in OTFT devices, respectively. Figure 3(a) shows the schematic representation of the realization of the P3HT-TFTs with BC configurations. P3HT pixels were transferred successfully at a laser fluence of $220 \mathrm{~mJ} / \mathrm{cm}^{2}$ between the previously printed electrodes. The printed pixels had a rectangular shape without any remarkable splashes or ejected particles around them. Figure 3(b) illustrates the quality of the laser-printed P3HT pixel in between the laser printed Ag-NPs S/D electrodes. Each laser pulse prints near-identical $270 \times 270 \mu \mathrm{m}^{2}$ size P3HT pixels. a)

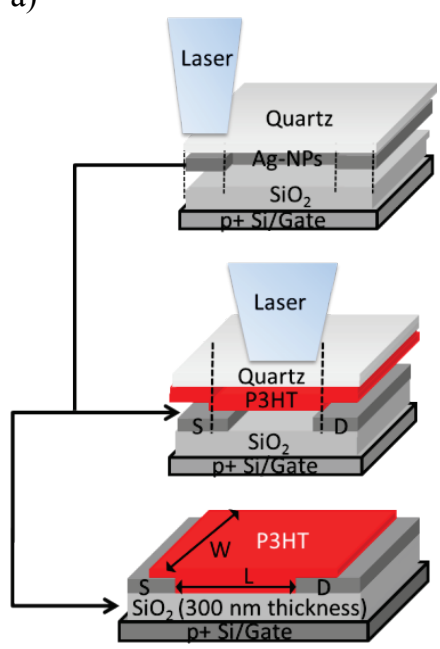

b)

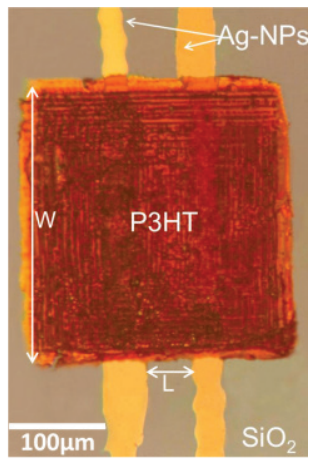

Fig. 3 (a) Schematic representation of the realization of organic TFTs with $\mathrm{BC}$ configurations. (b) Optical microscope image of a completed P3HT-TFT with a channel width (W) of $270 \mu \mathrm{m}$ and a channel length (L) of $50 \mu \mathrm{m}$.
Typical I-V output of P3HT-TFTs fabricated with printed Ag electrodes with $\mathrm{BC}$ configurations show that BC-TFTs exhibit linearity between $I_{D S}$ and $V_{D S}$ in low voltage regimes $(0$ to $-10 \mathrm{~V})$. All the $\mathrm{P} 3 \mathrm{HT}$-TFTs have a channel width of $\mathrm{W}=270 \mu \mathrm{m}$ and a channel length of $\mathrm{L}=10$ to 70 $\mu \mathrm{m}$. The values of the field-effect mobility $\left(\mu_{\mathrm{FET}}=3.7 \cdot 10^{-5}\right.$ $\left.\mathrm{cm}^{2} / \mathrm{Vs}\right)$ and the threshold voltage $\left(\mathrm{V}_{\mathrm{T}}=3 \mathrm{~V}\right)$ have been extracted from the related transfer curves at a $V_{D S}=-50 \mathrm{~V}$. All measurements were performed in air with the devices shielded from ambient light. More precisely, $\mu_{\mathrm{FET}}$ and $\mathrm{V}_{\mathrm{T}}$ were extracted from the transfer characteristics in the saturation regime. In the saturation regime, $I_{D S}$ is determined from the following equation:

$$
\mathrm{I}_{\mathrm{DS}}=\frac{\mathrm{W}}{2 \mathrm{~L}} \mu_{\mathrm{FET}} \mathrm{C}_{\mathrm{i}}\left(\mathrm{V}_{\mathrm{G}}-\mathrm{V}_{\mathrm{T}}\right)^{2}
$$

where $I_{D S}$ is the saturated drain current, $C_{i}$ is the dielectric capacitance per unit area and $\mathrm{V}_{\mathrm{G}}$ is the gate voltage.

One critical material for OTFTs is the channel semiconductor which dictates transistor performance. Poly(3,3"'-didodecylquarterthiophene) (PQT-12) is a novel high performance polymeric semiconductor belonging to one of the regioregular polythiophene systems. It has a high ionization potential $(0.1 \mathrm{eV}$ higher than that of a common organic semiconductor (P3HT)) and a wider band gap, indicative of its greater stability against photoinduced oxidative doping leading to the improvement of the performance of devices made from it. In this context, PQT12 based TFTs were realized with BC configuration as shown in Fig. 4. More specifically, a dry silicon dioxide layer $100 \mathrm{~nm}$ thick was grown on an implanted p-type Si substrate (receiver substrate) acting as the gate oxide and the gate electrode respectively. $\mathrm{Au} / \mathrm{Ti}$ metal pads were sputtered on the $\mathrm{SiO}_{2}$ layer through a shadow mask, forming the source and drain electrodes. The polymer material (PQT-12) was transferred from a donor substrate onto the receiver substrate covering the $\mathrm{S} / \mathrm{D}$ electrodes. The transfer of PQT-12 was achieved at low fluencies $(<100$ $\mathrm{mJ} / \mathrm{cm}^{2}$ ) by using an intermediate sacrificial layer (sulfonate-based triazene polymer). The output characteristics of PQT-12 based TFT devices which are presented in Fig. 4 (b), exhibited good saturation behavior indicating good performance of the OTFTs produced. Later experiments resulted in PQT-12 based TFTs with a high mobility $\left(\mu_{\mathrm{FET}}=2.3 \times 10^{-2} \mathrm{~cm}^{2} / \mathrm{Vs}\right)$ but a low ON/OFF ratio. In addition, the transfer of a multilayer stack of material in a single step LIFT process in order to realize OTFTs in the top gate configuration is under investigation. In this case, the donor substrate consists of multiple layers for transfer (gate material, organic dielectric, organic semiconductor) on to the receiver materials such as $\mathrm{Si}$ or plastic substrates. Initial transfers with an $\mathrm{Nd}$ :YAG laser look promising and show that the stacked layers from the donor substrates can be successfully transferred onto the receiver substrate.

To conclude, this work shows that operative OTFT devices can be fabricated using the LIFT technique. The broad range of materials that can be printed ranging from $\mathrm{Ag}$ nano-ink to organic semiconductors confirms the potential of LIFT as a very versatile technique. 
a)
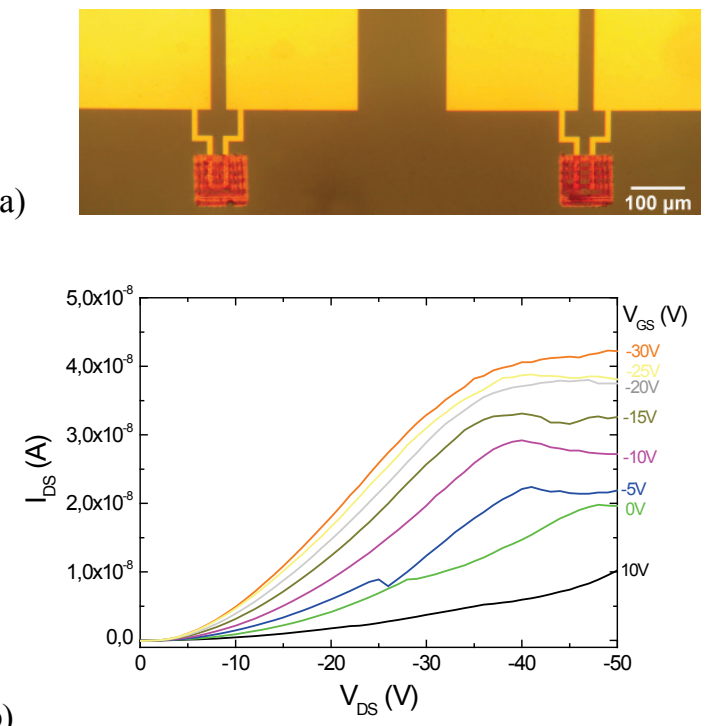

Fig. 4 (a) Optical microscope image of a completed PQT-TFT with a channel width of $100 \mu \mathrm{m}$ and a channel length of $40 \mu \mathrm{m}$. (b) Output characteristics: $\mathrm{V}_{\mathrm{GS}}$ was varied between $10 \mathrm{~V}$ and $-30 \mathrm{~V}$.

\subsection{Sensors}

LIFT process was also used as an advanced tool for the immobilization of photosynthetic materials onto nonfunctionalized SPEs and for the deposition of oligonucleotides on the surfaces of ultrathin low temperature oxide (LTO) membranes.

The photosynthetic materials were thylakoid membranes, extracted from Spinacea oleracea and the direct immobilization of the thylakoids was confirmed by amperometry.

2D patterns of discrete thylakoid droplets or continuous layers were printed on the electrodes by adjusting droplets separation distance. The transfer was carried out in such a way that each droplet was deposited by a single pulse and the optimum distance between the target and the substrate was found to be $300 \mu \mathrm{m}$. Following the optimization study, the laser transfer was performed at $470 \mathrm{~mJ} / \mathrm{cm}^{2}$ and the laser beam size on the donor substrate was $60 \mu \mathrm{m}$ in diameter. By using these laser parameters the deposited thylakoid droplets on the electrode surface were $150 \mu \mathrm{m}$ in diameter.

The measurement of the Photosystem II (PSII) activity was based on amperometric registration of the reduced form of artificial electron acceptors under a specific potential. Fig. 5 shows typical electrical activity signals of gold SPEs printed by using LIFT and the reference pipette spotting method. The laser printed gold SPEs present increased photocurrent activity (approaching a factor of 10 better) indicating strong immobilization of the laser-printed photosynthetic material onto the gold electrode surface without the use of linkers. Moreover, the high electrical signal is combined with an extremely high signal-to-noise ratio. For comparison, the same thylakoid material was also deposited using a pipette and the measured current peaks were very low indicating weak immobilization of the photosynthetic material on the gold electrode surface.
Laser printing is an excellent tool for both printing and immobilization of the photosynthetic material on the gold SPEs surface. The explanation for this phenomenon is given by investigating the physical aspects of the LIFT process and the high roughness surface of the gold SPEs, which is of the order of $\mu \mathrm{m}$. Surfaces with protrusions present two wetting states, namely the Cassie state (partial wetting) and the Wenzel state (complete wetting). It has been reported that an applied pressure on a drop (e.g manually applied force, high speed projectiles, etc) can force the liquid into the protrusions between the projectiles, and thus transform the Cassie state into the Wenzel state resulting in complete wetting of a rough surface [10]. As a result of this wetting state transition, physical adsorption of thylakoid membranes on the electrode surface is enhanced resulting in high activity signals. Similar results were also obtained for LIFT-printed carbon nanotubes and graphite SPEs.

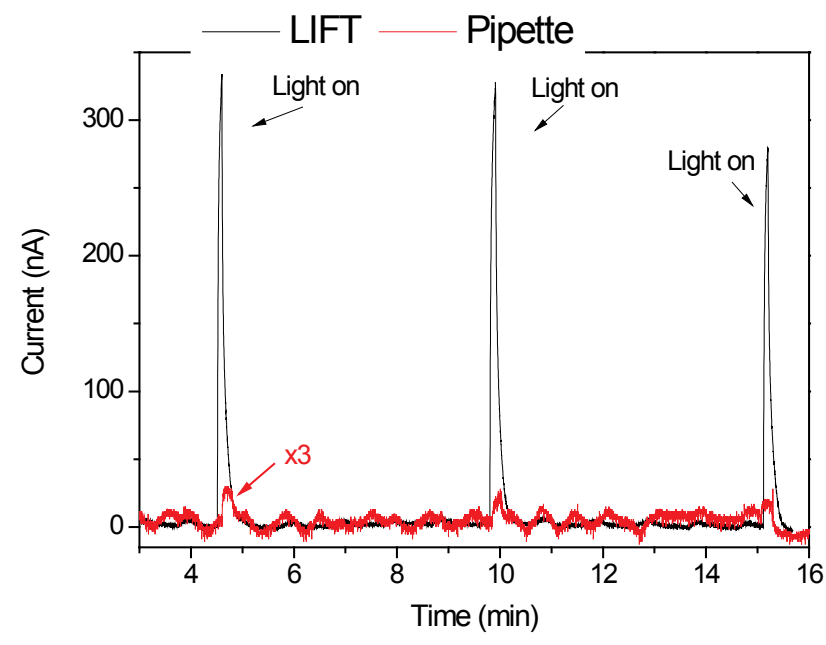

Fig. 5 Comparison between typical electrical signals for electrodes fabricated by: LIFT (black line, exhibiting currents up to $340 \mathrm{nA}$, and pipette deposited (red line exhibiting current values up to $40 \mathrm{nA}$ ).

For the fabrication of a capacitance biosensor, three different 15-mer DNA probe sequences were printed on the surface of ultra-thin LTO membranes. The first one was fully complementary to the target analyte in solution. The second one formed one internal mismatch base-pair with the same target sequence, while the third probe was not complementary to the target analyte and was used as a reference. For the functionalization of the substrate, a monolayer of 3-glycidoxypropyl tri-methoxy silane (GOPTS) was fabricated onto the surface of the LTO. The synthesized oligonucleotides were chemically modified by the addition of a thiol group (-SH) at their 5'end to be able to form covalent bonds with the silane coating used.

The thickness of each membrane was $1 \mu \mathrm{m}$ LTO on $\mathrm{Si}$, and LIFT enabled the deposition of the oligonucleotide solutions on the surface of the above membranes, without the risk of destroying their flexibility. Each sensor array consisted of 60 sensing elements (membranes) and reference elements (4 $\mathrm{Al}$ capacitors). 
In Fig. 6 it can be seen that the LIFT technique also allowed the coverage of a substantial percentage of the membrane and the ability to cover each membrane with a different oligonucleotide solution, due to the high spatial resolution of the technique.

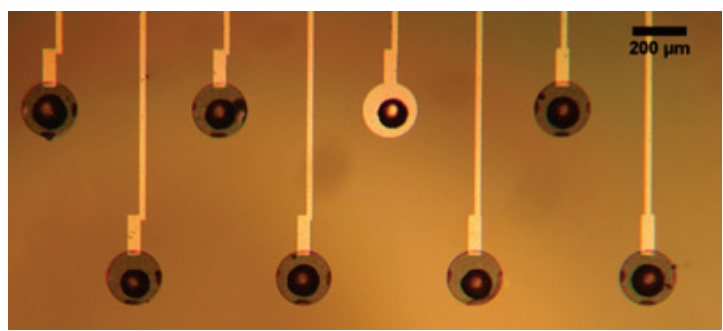

Fig. 6 Optical microscopy image of the laser printed droplets on the ultra-thin membranes of the capacitance biosensor.

\section{Conclusion}

In conclusion, various applications of LIFT are presented in this manuscript. It is proved that LIFT is a very versatile printing technique due to the fact that a broad range of materials ranging from $\mathrm{Ag}$ nano-ink to organic semiconductors can be printed. Examples of electronic devices include the printing of $\mathrm{Ag}$ lines and the operative P3HT-based Thin Film Transistors with bottom contact configuration on silicon or flexible substrates are demonstrated.

Additionally, the laser printing of biomaterials such as thylakoid membranes and oligonucleotides for the fabrication of biosensors, proves that LIFT is an advancing tool for biotechnological applications.

\section{Acknowledgments}

Financial support from European Commission ICT: e-LIFT, Grant Agreement No. 247868 is gratefully acknowledged. The author would like to acknowledge the invaluable help of Prof. Rob Eason for thoroughly reviewing the manuscript.

\section{References}

[1] S. Gamerith, A. Klug, H. Scheiber, U. Scherf, E. Moderegger, E.J.W. List, "Direct Ink-Jet Printing of Ag$\mathrm{Cu}$ Nanoparticle and Ag-Precursor Based Electrodes for OFET Applications", Adv. Funct. Mater. 17, 3111-3118 (2007).

[2] Y. Noguchi, T. Sekitani, T. Yokota, T. Someya, "Direct inkjet printing of silver electrodes on organic semiconductors for thin-film transistors with top contact geometry", Appl. Phys. Lett. 93, 043303 (2008).

[3] L. Barthelmebs, R. Carpentier, and R. Rouillon, "Physical and Chemical Immobilization Methods of Photosynthetic Materials', Methods Mol. Biol. 684, 247-256 (2011).

[4] K. Buonasera, G. Pezzotti, V. Scognamiglio, A. Tibuzzi, M. T. Giardi, "New Platform of Biosensors for Prescreening of Pesticide Residues To Support Laboratory Analyses', J. Agric. Food Chem. 58, 5982-5990 (2010).

[5] C. B. Arnold, P. Serra, and A. Pique, "Laser directwrite techniques for printing of complex materials', MRS Bull. 32, 23-31 (2007).
[6] M. Duocastella, J.M. Fernandez-Pradas, P. Serra, J.L. Morenza "Jet formation in the laser forward transfer of liquids”, Appl. Phys. A: Mater. Sci. Process. 93, 453-456 (2008).

[7] P. C. Duineveld "The stability of ink-jet printed lines of liquid with zero receding contact angle on a homogeneous substrate" J. Fluid Mech. 477, 175-200 (2003).

[8] H. Kim, R. C. Y. Auyeung, S. H. Lee, A. L. Huston, A. Piqué, "Laser-printed interdigitated $\mathrm{Ag}$ electrodes for organic thin film transistors", J. Phys. D: Appl. Phys. 43, 085101 (2010).

[9] L. Rapp, A. K. Diallo, A. P. Alloncle, C. VidelotAckermann, F. Fages, P. Delaporte, "Pulsed-laser printing of organic thin-film transistors", Appl. Phys. Lett. 95, 171109 (2009).

[10] B. Liu and F. F. Lange, "Wetting transition of water droplets on superhydrophobic patterned surfaces" J. Colloid Interface Sci. 298, 899 (2006).

(Received: June 27, 2012, Accepted: November 15, 2012) 